\title{
TWO-PARTICLE PARASITIC EFFECTS IN RARE-EARTH DOPED WAVEGUIDES
}

\author{
B. JASKORZYNSKA* \\ Royal Institute of Technology, Dept. of Electronics \\ Electrum 229, 16440 Kista, Sweden
}

\begin{abstract}
This paper discusses Er-doped optical waveguides implemented in glass materials. The emphasis is put on physical limitations posed by concentration dependent nonlinear effects and on methods for their characterization. Examples of recently demonstrated, best performance integrated active devices are also given.
\end{abstract}

PACS numbers: $42.65,-\mathrm{k}$

Optical fibers and planar waveguides can be made active, i.e. exhibit amplification, by inserting rare-earth ions in their core. Most of the related research has been focused on fibers and planar waveguides doped with erbium (Er) because. they provide gain at the $1.5 \mu \mathrm{m}$ band, which is of particular interest for telecommunication applications. For pumping Er ions one typically uses laser diodes at $980 \mathrm{~nm}$ or $1.48 \mu \mathrm{m}$, as it is illustrated in Fig. 1 .

Planar active waveguides are considered to be an attractive option for realizing integrated optical devices with gain $[1,2]$. A prerequisite for realizing practical active devices is that a sufficiently large gain can be obtained at reasonable pumping powers. While this can easily be achieved in a few meter long active fibers, their planar counterparts tend to suffer from low gain efficiencies. Apart from much higher losses, the main difficulty is that a usable gain must be accumulated over a much shorter (a few centimeters) length. This. typically requires two orders of magnitude higher $\mathrm{Er}^{3+}$ concentrations, at which parasitic energy transfer between excited Er-ions reduces the gain [3-10]. The mechanism of the parasitic effect is illustrated in Fig. 2. The excitation energy (1) is transferred (2) from one excited ion (donor) to the other (acceptor), which typically occurs via electric dipole-dipole interaction. Consequently the donor relaxes (2) to the ground state while the acceptor is upconverted to the ${ }^{4} I_{9 / 2}$ state (2). This is usually followed by two fast, non-radiative relaxation processes $(\mathbf{3 , 4 a})$ down to the metastable level ${ }^{4} I_{13 / 2}$. However, a small fraction of the ions relaxes to the ground level $(4 \mathbf{b})$ emitting photons at $980 \mathrm{~nm}$. As a result only one of the two pump photons absorbed by the Er ions can be used for amplification at $1.5 \mu \mathrm{m}$. The energy of the other

*e-mail: bj@ele.kth.se 

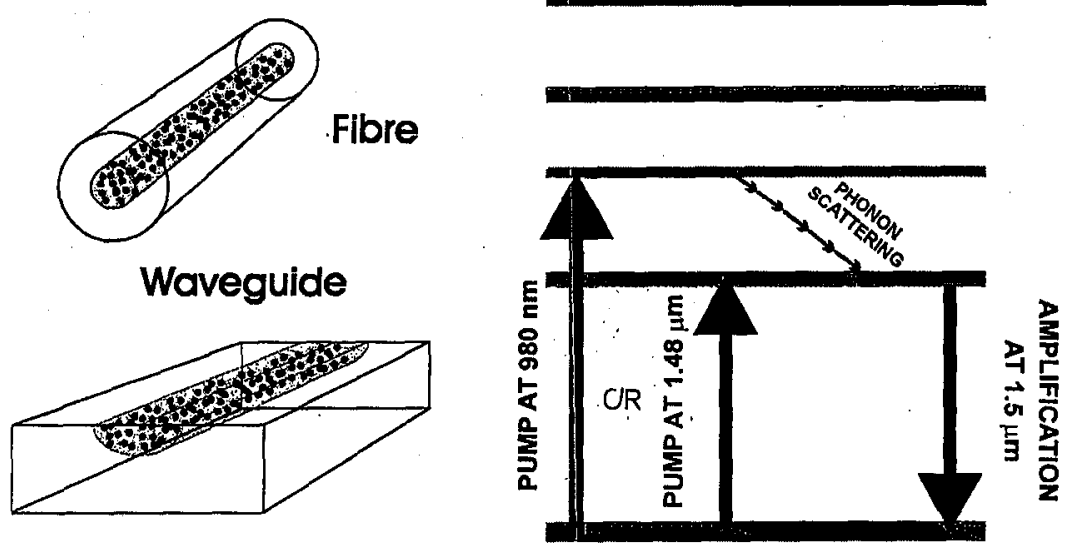

Fig. 1. Amplification in Er-doped fibers and planar waveguides.

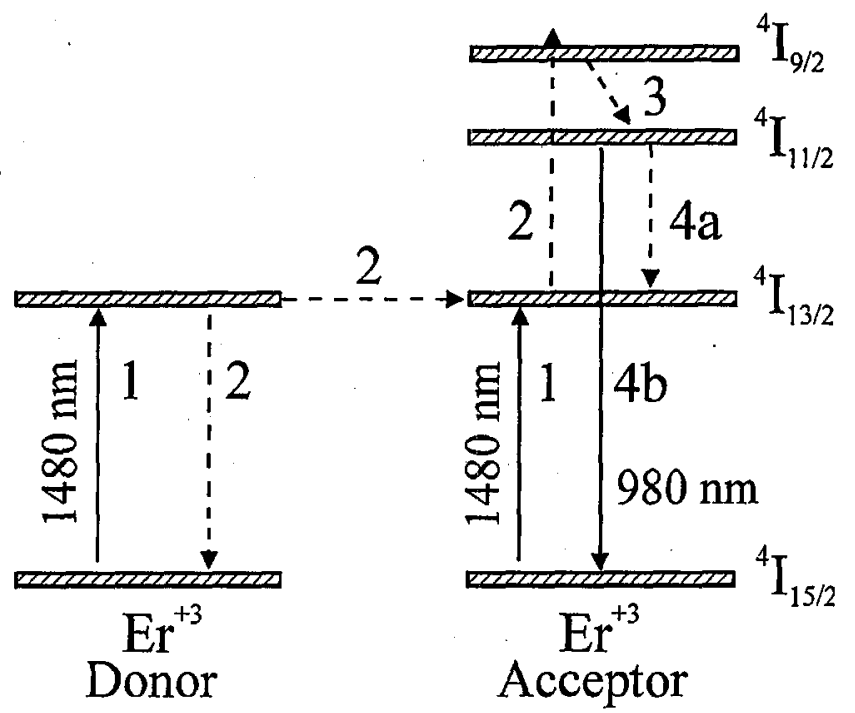

Fig. 2. Two-particle upconversion between $\mathrm{Er}^{3+}$ ions. Dashed arrows represent non-radiative transitions.

one is lost for phonons or $980 \mathrm{~nm}$ fluorescence: The power in the upconverted fluorescence at $980 \mathrm{~nm}$ is proportional to the rate of the upconversion process.

For randomly distributed ions the process is called uniform or homogeneous upconversion (HUC). It causes gain reduction that can be compensated by increasing pumping power, as it can be seen in Fig. 3a. HUC scales roughly with the square of the Er concentration and it sets an ultimate limit on the gain efficiency. Nevertheless, for many applications a sufficient performance can still be achieved even in a very short $(<1 \mathrm{~cm})$ active device. For clustered ions, however, energy 

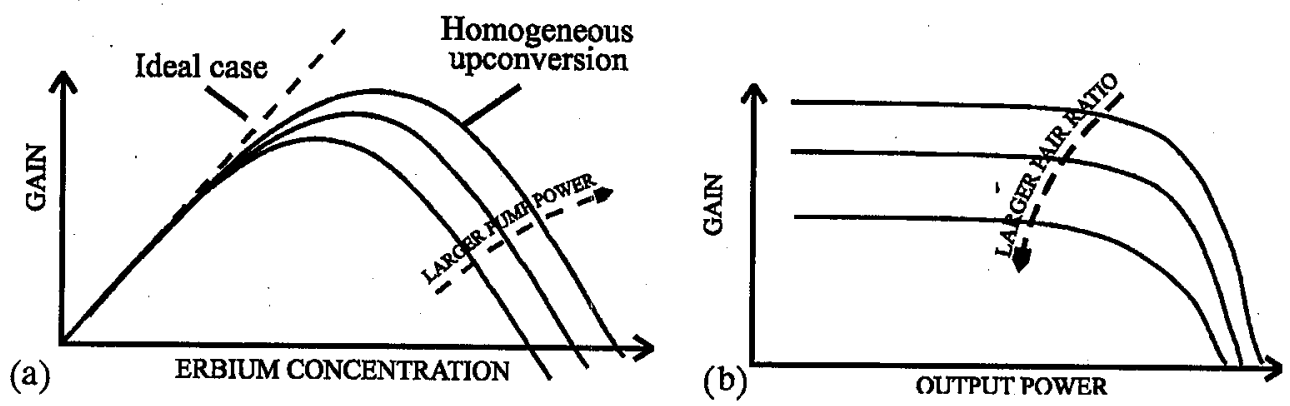

Fig. 3. Gain reduction with increasing Er concentration is smaller for larger pump powers (a). Gain reduction with increasing ratio of clustered Er ions (b).

transfer is several orders of magnitude faster [3], and therefore, the gain reduction cannot be compensated at any practical levels of pumping powers. All but one ion in a cluster effectively stay in the ground state, which for a three-level system implies that the gain from clustered ions is totally quenched. This effect is called pair induced quenching (PIQ). The reduction of the gain with increasing ratio of clusters (pairs) is illustrated by Fig. $3 \mathrm{~b}$.

Clustering of ions can be minimized by choice of a host material composition or fabrication parameters. Distinction and quantitative evaluation of the two concentration dependent processes is essential for an assessment of applied fabrication techniques and optimizing design of densely doped devices.

A number of methods that have been developed for optical fibers can be either directly applied, or modified, to investigate the parasitic effects in planar waveguides.

This includes the methods based on analysing steady-state $[9,10]$ or dynamic $[3,6,10]$ characteristics of upconverted fluorescence, and on measurements of non-saturable absorption [6, 11, 12]. The recently observed [13] difference in absorption spectra of clustered and randomly distributed ions may be used as an additional technique for clustering diagnosis.

One way to check the presence of clusters is to measure steady-state fluorescence at $980 \mathrm{~nm}$ and $1.5 \mu \mathrm{m}$ for increasing pump powers and check if they are related by the square low. The upconverted fluorescence caused by HUC is proportional to the square of the inverted population $N_{2}$ while $1.5 \mu \mathrm{m}$ fluorescence is directly proportional to $N_{2}$. Deviations from the quadratic dependence between the powers at $980 \mathrm{~nm}$ and $1.5 \mu \mathrm{m}$ can be attributed to the effect of clusters (PIQ). This is illustrated in Fig. 4.

The most direct method for quantitative estimation of the clustering relies on evaluation of the non-saturable absorption effect. Since only one of the clustered ions can be effectively inverted, all the others will absorb the pump power no matter how strong the pumping is. Therefore, the pump transmission can never reach one in the presence of clusters. The larger is portion of clusters the smaller are the values of the maximum transmission, as it is shown in Fig. 5.

The clustering problem is related to solubility of rare-earth ions in a host material. For low solubility a long waveguide path is needed in order to minimize 


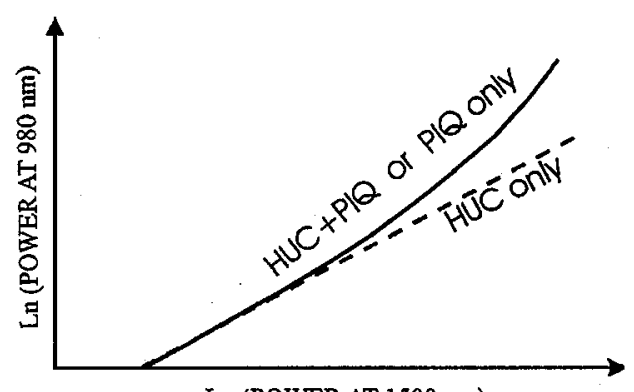

Fig. 4.

Ln (POWER AT $1500 \mathrm{~nm}$ )

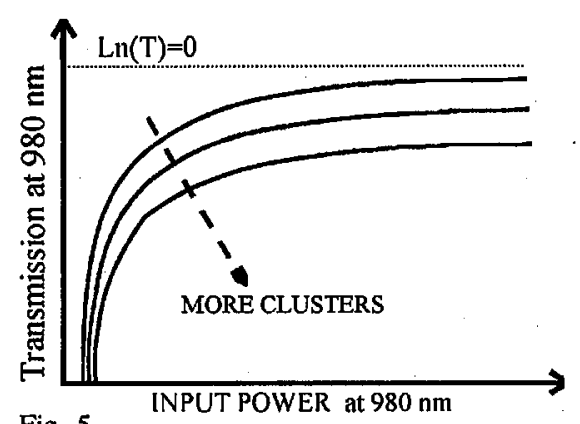

Fig. 5.

Fig. 4. Logarithmic plot of powers in $980 \mathrm{~nm}$ and $1.5 \mu \mathrm{m}$ fluorescence for increasing pump power. The linear part of the curve corresponds to the quadratic dependence.

Fig. 5. Effect of non-saturable absorption for increasing amount of clusters.

clustering. Although the waveguide can be bent to keep the device area smaller, as it was demonstrated for Er-doped silica glass [14], such a solution requires a high refractive index contrast to minimize bending losses, and also limits complexity of integration with passive devices. Therefore, a gain-per-centimeter length is often used as a relevant figure of merit for planar waveguides. However, the gain efficiency, i.e. the total gain per $\mathrm{mW}$ of a pump power, should not be forgotten in the assessment of the device performance.

In soda lime glass and phosphate glass Er has a high solubility with a low degree of clustering. High performance active waveguides have recently been reported in those materials:

$4.2 \mathrm{~dB} / \mathrm{cm}$ gain with $7.2 \mathrm{~dB}$ total gain at $40 \mathrm{~mW}$ pump power - soda lime [15], $4.1 \mathrm{~dB} / \mathrm{cm}$ gain, with $4.1 \mathrm{~dB}$ total gain at $21 \mathrm{~mW}$ pump power - phosphate [16]. By codoping Er-waveguides with ytterbium $(\mathrm{Yb})$ one can benefit from the fact that $\mathrm{Yb}$ ions have a much larger pump absorption cross-section than Er. Provided energy transfer from $\mathrm{Yb}$ to $\mathrm{Er}$ ions is very efficient, the pump power can be better utilized. Moreover, the presence of $\mathrm{Yb}$ decreases tendency of $\mathrm{Er}$ ions for clustering. For those reasons one can expect a high gain efficiency in $\mathrm{Yb} / \mathrm{Er}$-doped waveguides $[17,18]$. This is confirmed by the recent demonstrations of high-performance laser [19], amplifier [20] and an amplifying four-wavelength combiner [21]. A pigtailed amplifier module has been realized in an $\mathrm{Er} / \mathrm{Yb}$-doped phosphate glass and successfully used as a booster amplifier in $10 \mathrm{~Gb} / \mathrm{s}$ system experiment [22].

In conclusion: concentration dependent parasitic effects, homogeneous upconversion and pair induced quenching caused by ion clustering, reduce gain in densel'y doped rare-earth devices. HUC that reduces the gain efficiency is unavoidable and hence imposes a fundamental limit on the device performance. However; PIQ that may totally quench the gain can be eliminated or minimized in the fabrication process. A number of optical methods have been developed to distinguish between the two effects.

The recent advances clearly show that planar active waveguides approach the performance determined by the fundamental limitations, and that integrated active devices are becoming a realistic option for numerous system applications. 


\section{References}

[1] D. Barbier, in: Optical Amplifiers and their Applications, in series OSA Trends in Optics and Photonics, Vol. 16, Eds. M.N. Zervas, A.E. Willner, S. Sasaki, Optical Society of America, Washington (DC) 1997, p. 72.

[2] J. Shmulovich, in: Rare-Earth-Doped Devices, Ed. S. Honkanen, in series Proc. SPIE, Vol. 2996, 1997, p. 143.

[3] P. Myslinski, J. Fraser, J. Chrostowski, Topical Meeting on Optical Amplifiers and their Asplications, OSA Technical Digest, Optical Society of America, Washington (DC) 1995 , p. 100.

[4] P. Blixt, J. Nilsson, T. Carlnäs, B. Jaskorzynska, IEEE Photonics Technol. Lett. 3, 996 (1991).

[5] J. Nilsson. B. Jaskorzynska, P. Blixt, IEEE Photonics Technol. Lett. 5, 1427 (1993).

[6] P. Myslinski, Dung Nguyen, J. Chrostowski, J. Lightwave Technol. 15, 112 (1997).

[7] T. Ohtsuki, S. Honkanen, S.I. Najafi, N. Peyghambarian, J. Opt. Soc. Am. B 14, 1838 (1997).

[8] M.P. Hehlen, N.J. Cockroft, T.R. Gosnell, A.J. Bruce, G. Nykolak, J. Shmulovich, Opt. Lett. 22, 772 (1997).

[9] R.S. Quimby, W.J. Miniscalco, B. Thompson, J. Appl. Phys. 76, 4472 (1994).

[10] J. Nilsson, P. Blixt, B. Jaskorzynska, J. Babonas, J. Lightwave Technol. 13, 341 (1995).

[11] M.K. Davis, M.J.F. Digonnet, R.H. Pantell, J. Lightwave Technol. 13, 120 (1995).

[12] C.C. Ye, B. Jaskorzynska, S. Helmfrid, P.-Y. Fonjallaz, Appl. Opt. 37, 6362 (1998).

[13] B.N. Samson, W.H. Loh, J.P. de Sandro, Opt. Lett. 22, 1763 (1997).

[14] K. Hattori, T. Kitagawa, M. Oguma, Y. Ohmori, M. Horiguchi, Electron. Lett. 30, 856 (1994).

[15] Cheng-Chung Li, Hong-Koo Kim, M. Migliuolo, IEEE Photonics Technol. Lett. 9, 1223 (1997).

[16] Y.C. Yan, A.J. Faber, H. de Waal, P.G. Kik, A. Polman, Appl. Phys. Lett. 71, 2922 (1997).

[17] J. Nilsson, P. Scheer, B. Jaskorzynska, IEEE Photonics Technol. Lett. 6, 383 (1994).

[18] F. Di Pascuale, M. Federighi, IEEE J. Quantum Electron. 30, 2127 (1994).

[19] A. Yeniay, J.M.P. Delavaux, J. Toulouse, D. Barbier, T.A. Strasser, J.R. Pedrazanni, IEEE Photonics Technol. Lett. 9, 1099 (1997).

[20] P. Fournier, P. Meshkinfam, M.A. Fardad, M.P. Andrews, S.I. Najafi, Electron. Lett. 33, 293 (1997).

[21] D. Barbier, M: Rattay, F. Saint André, G. Clauss, M. Trouillon, A. Kevorkian, J.-M.P. Delavaux, E. Murphy, IEEE Photonics Technol. Lett. 9, 315 (1997).

[22] J.M.P. Delavaux, S. Granlund, O. Mizuhara, L.D. Tzeng, D. Barbier, M. Rattay, F. Saint André, A. Kevorkian, IEEE Photonics Technol. Lett. 9, 247 (1997). 